\title{
Diagnostic Value of Serum Biomarkers for Patients Undergoing Curative Resection with Non-B, Non-C Hepatocellular Carcinoma
}

\author{
Youdi Li1, Yanfei Chen² and Jiu Chen 1 \\ ${ }^{1}$ Department of Traditional Chinese Medicine, The First Affiliated Hospital, College of Medicine, Zhejiang University, Hangzhou, China \\ ${ }^{2}$ State Key Laboratory for Diagnosis and Treatment of Infectious Diseases, Collaborative Innovation Center for Diagnosis and \\ Treatment of Infectious Diseases, The First Affiliated Hospital, College of Medicine, Zhejiang University, Hangzhou, China
}

\begin{abstract}
Objective: To determine the diagnostic value of alpha-fetoprotein (AFP) and des-gamma-carboxy prothrombin (DCP) for patients with non-B, non-C hepatocellular carcinoma (NBNC HCC).

Study Design: Observational study.

Place and Duration of Study: The First Affiliated Hospital, College of Medicine, Zhejiang University, Hangzhou, China, from June 2016 to June 2019.

Methodology: Patients with NBNC HCC diagnosed by histology were inducted after curative resection. Serum levels of AFP and DCP were determined. Their receiver operating characteristic (ROC) curves were generated, and the area under the ROC curve (AUC) calculated. In subgroup analyses involving patients with hepatitis $B$ core antibody (HBcAb) testing, the AUC of AFP and DCP were also calculated and compared.

Results: Serum levels of AFP and DCP were clearly increased in the NBNC HCC group relative to controls ( $p=0.000$ for both). The AUC for AFP, DCP and their combination (AFP+DCP) were 0.763 (95\%Cl: 0.712-0.814), 0.853 (95\%Cl: 0.811-0.894), and 0.867 (95\% $\mathrm{Cl}$ : 0.829-0.906), respectively. For HBcAb-positivity, the AUC of AFP and DCP were $0.7517(95 \% \mathrm{Cl}$ : $0.6917-0.8116)$ and 0.8469 (95\% Cl: 0.7985-0.8963), respectively. For HBcAb-negativity, the AUC of AFP and DCP were 0.8083 (95\%Cl: $0.6993-0.9174)$ and 0.8740 (95\% Cl: 0.7901-0.9580), respectively.

Conclusion: A combination of AFP and DCP is useful for assisting the diagnosis of NBNC HCC before surgery. AFP and DCP showed better diagnostic accuracy for HBcAb-negativity compared with HBcAb-positivity in NBNC HCC patients.
\end{abstract}

Key Words: Diagnosis, Alpha-fetoprotein, Des-gamma-carboxy prothrombin, Non-B, Non-C hepatocellular carcinoma, Hepatitis B core antibody.

How to cite this article: Li Y, Chen Y, Chen J. Diagnostic value of serum biomarkers for patients undergoing curative resection with non-B, non-C hepatocellular carcinoma. J Coll Physicians Surg Pak 2020; 30(2):134-138.

\section{INTRODUCTION}

The morbidity of virus-related hepatocellular carcinoma (HCC) has been decreasing worldwide because of vaccination, antiviral therapy, and screening of transfused blood.1-3 Nevertheless, HCC is still one of the main leading causes of cancer-related death worldwide. ${ }^{4}$ One reason may be that the morbidity of non-B, non-C (NBNC) HCC, which is negative for the hepatitis- $B$ surface antigen ( $\mathrm{HBsAg}$ ) and hepatitis-C (HCV) antibody, has been increasing continuously. ${ }^{5-7}$ One survey revealed that the proportion of NBNC HCC accounted for $32.5 \%$ of all HCC cases in Japan in 2015; whereas, the proportion was only $10 \%$ in 1991.7

NBNC HCC carries a poor prognosis. ${ }^{4}$ Early diagnosis and curative resection are the basis for successful treatment. ${ }^{8}$ Without the alert of virus markers, most NBNC

Correspondence to: Dr. Jiu Chen, Department of Traditional Chinese Medicine, The First Affiliated Hospital, College of Medicine, Zhejiang University, Hangzhou, China

E-mail: linyunju@zju.edu.cn

Received: October 10, 2019; Revised: December 28, 2019;

Accepted: January 13, 2020
HCC cases do not have periodic medical examinations and are diagnosed at an advanced stage.5,9 Ultrasonography (US), computed tomography (CT) and magnetic resonance (MR) have been used for diagnosis, but these techniques are dependent on the quality of the equipment and expertise of the operator. Some people could not be examined for their own reasons, such as allergy to the contrast. Thus, regular HCC surveillance, as performed for patients with viral chronic liver disease and diagnostic markers are required for simple, specific and rapid detection of NBNC HCC. 10

Alpha-fetoprotein (AFP) is used widely for the diagnosis of $\mathrm{HCC}$, but $\sim 30 \%$ of early-stage HCC cases cannot be detected only by using AFP measurement. 11 The diagnostic value of using des-gamma-carboxy prothrombin (DCP) in HCC has been studied, but has been inconsistent because of different backgrounds of liver disease. 12

The morbidity of NBNC HCC is continually increasing worldwide without reliable and readily accessible biomarkers ${ }^{5-7}$ and the diagnostic value of serum AFP and DCP has seldom been studied for NBNC HCC. 
The aim of this study was to determine the diagnostic value of serum levels of AFP and DCP and their combination for NBNC HCC in this study.

\section{METHODOLOGY}

All the participants were enrolled retrospectively from June 2016 to July 2019 at The First Affiliated Hospital, College of Medicine, Zhejiang University (Hangzhou, China). All participants were NBNC HCC patients aged $>18$ years who had surgical resection and were diagnosed by histology after surgery. Ethics approval protocol was obtained from the Ethics Committees of the Hospital, for this research.

The exclusion criteria were patients taking warfarin or vitamin $\mathrm{K}$ within 2 weeks of blood-sample collection; who had undergone liver transplantation; in whom lesions in the liver could not be confirmed (e.g., people who refused surgery or biopsy); who exhibited evidence of malignancies other than NBNC HCC; and who received anti-cancer treatment before measurement of serum levels of biomarkers.

Serum AFP levels were tested by an automatic analyser (i2000SR; Abbott Diagnostics, Chicago, IL, USA) and associated kits. Serum DCP levels were tested by a chemiluminescent enzyme immunoassay (Lumipulse ${ }^{\circledR}$ G PIVKA-II; Fujirebio, Tokyo, Japan). SPSS 22.0 (IBM, Armonk, NY, USA) and Prism 5 (GraphPad, San Diego, $C A$, USA) were used for analyses. Statistical significance for tests was set at $p<0.01$. If quantitative data did not have a normal distribution, Mann-Whitney U-test was used to evaluate intergroup differences. To assess the diagnostic value of NBNC HCC, analyses of receiver operating characteristic (ROC) curves were conducted and the areas under the receiver operating characteristic curve (AUC) were calculated.

\section{RESULTS}

The characteristics of participants with NBNC HCC are listed in Table I. One hundred and thirty-six NBNC HCC patients and 259 cases of healthy controls were assigned to NBNC HCC group and control group, respectively. The normal ranges of DCP and AFP were $0-40 \mathrm{mAU} / \mathrm{ml}$ and $0-20 \mathrm{ng} / \mathrm{ml}$. Levels of AFP and DCP were measured and compared in the control group and NBNC HCC group. The mean levels of AFP and DCP were $615.2 \mathrm{ng} / \mathrm{ml}$ and $3247.03 \mathrm{mAU} / \mathrm{ml}$, respectively. Levels of AFP and DCP were clearly increased in the NBNC HCC group relative to controls, with $p$-values of 0.000 and 0.000 , respectively (Figure 1 ).

ROC curves were plotted to determine the diagnostic value of different serum biomarkers for NBNC HCC patients. AFP and DCP showed diagnostic accuracy with an AUC value of 0.763 and 0.853 , respectively; whereas, combination of AFP and DCP demonstrated the best diagnostic accuracy (AUC $=0.867$, Figure 2). Moreover,
DCP showed better diagnostic accuracy than AFP and compared with use of a single biomarker, use of a combination of biomarkers increased the diagnostic accuracy.

The positivity and negativity of hepatitis $B$ core antibody $(\mathrm{HBcAb})$ in NBNC HCC patients and controls were also explored respectively (Figure 3 ). Compared with HBcAbpositive NBNC HCC, both AFP and DCP showed better diagnostic accuracy for HBcAb-negative NBNC HCC.

\section{DISCUSSION}

We focused primarily on the accuracy of using measurements of AFP, DCP and their combination (AFP + DCP)

Table I: Characteristics of NBNC HCC patients..

\begin{tabular}{lc}
\hline Characteristics & Number of patients \\
\hline Age & \\
$\geq 50$ years & $93.38 \%(127)$ \\
$<50$ years & $6.62 \%(9)$ \\
\hline Gender & \\
Male & $80.88 \%(110)$ \\
Female & $19.12 \%(26)$ \\
\hline Positivity HBcAb & \\
Yes & $80.15 \%(109)$ \\
No & $19.85 \%(9)$ \\
\hline Hypertension & \\
Yes & $38.24 \%(52)$ \\
No & $61.76 \%(84)$ \\
\hline Type 2 diabetes & \\
Yes & $19.85 \%(27)$ \\
No & $80.15 \%(109)$ \\
\hline Tumor Number & \\
Single & $80.88 \%(110)$ \\
Multiple & $19.12 \%(26)$ \\
\hline Cirrhosis & \\
Yes & $38.24 \%(52)$ \\
No & $61.76 \%(84)$ \\
\hline Fatty liver & \\
Yes & $15.44 \%(21)$ \\
No & $84.56 \%(115)$ \\
\hline Schistosomal hepatopathy & \\
Yes & $5.88 \%(8)$ \\
No & $94.12 \%(128)$ \\
\hline Alcoholic liver & \\
Yes & \\
No & \\
\hline HBcAb: Hpatitis B core antibody & \\
& \\
\hline & \\
\hline
\end{tabular}

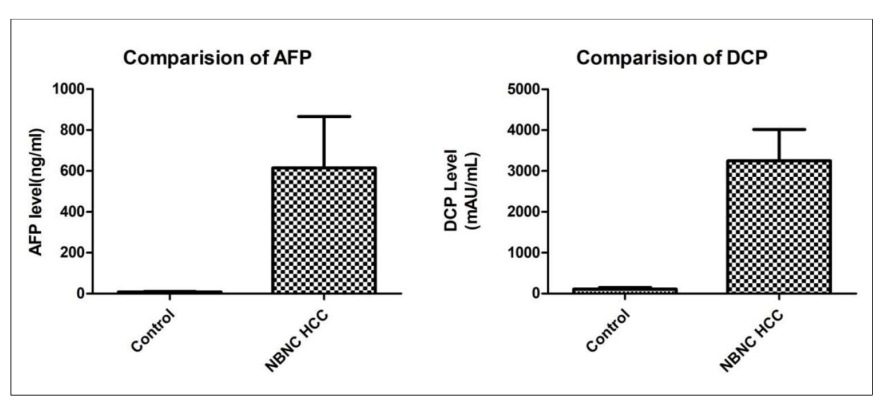

Figure 1: Comparison of levels of AFP and DCP in the control group and NBNC HCC group. 
for the diagnosis of NBNC HCC. The diagnostic accuracy of using AFP and DCP for HBcAb-negative and HBcAbpositive patients with NBNC HCC was also explored.

$\mathrm{HCC}$ is a common malignancy in Asia and the third most common cause of cancer-related death worldwide, only behind lung cancer and gastric cancer. ${ }^{4}$ Although most

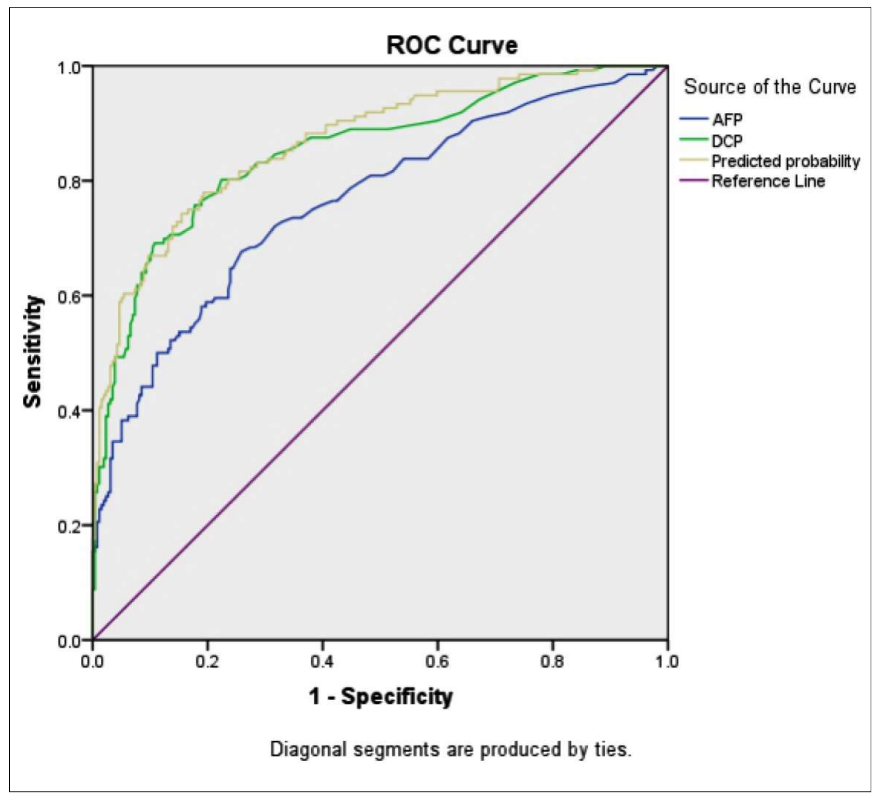

Figure 2: ROC curves of AFP, DCP, and AFP+ DCP.

ROC curve of AFP in positivity of HBCAb
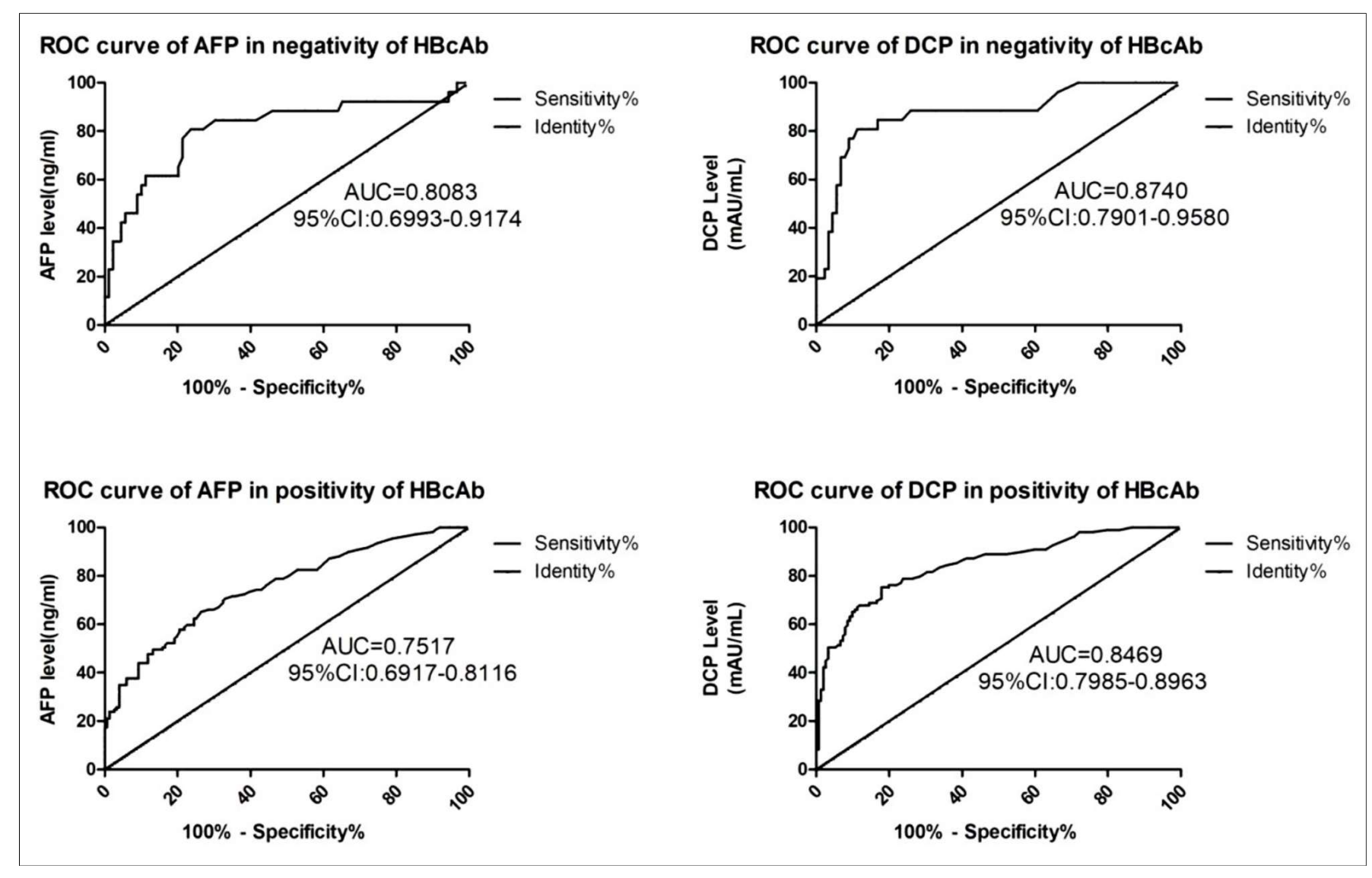

$\mathrm{HCC}$ is related to viral infection, the incidence of NBNCHCC has recently tended to increase.6-8 The exact aetiologies of NBNC HCC is still unclear. Non-alcoholic fatty liver disease (NAFLD) and non-alcoholic steatohepatitis (NASH) were considered to progress to cirrhosis and NBNC HCC. 5 High-fat diet and alcohol were the two major risk factors responsible for metabolic diseases; and NBNC HCC was considered to be one kind of the manifestation. ${ }^{13}$ But, other studies found that alcohol use and any other identifiable cause were not found in up to $38.0 \%$ of the NBNC HCC patients. ${ }^{14}$ In this study, it is found that the proportion of HBcAbpositive was up to $80 \%$ in all NBNC HCC cases. The fatty liver and alcohol liver were less than $20 \%$ in the NBNC HCC cases and the proportion of cirrhosis was less than $40 \%$. NBNC HCC was more likely to happen in $\mathrm{HBcAb}$-positive old male patients and be presented as one single lesion (average diameter of $4.66 \mathrm{~cm}$ ).

US, CT and MR have been used for diagnosis with different diagnostic accuracy; among them, gadoxetic acid-enhanced MRI was the most expensive technique with the highest diagnostic accuracy. But some patients could not be examined by gadoxetic acid-enhanced MRI because of allergy, renal insufficiency, metal stents in the body and so on. All these techniques are dependent on the quality of the equipment and expertise of the operator. In this study, all patients were examined by CT,

Figure 3: ROC curves of AFP and DCP in HBcAb-positive and HBcAb-negative patients. 
MRI, or both before surgery. These are not routine physical examination items now in our country. The incidence of NBNC HCC is increasing worldwide, and reliable serum biomarkers of high diagnostic accuracy are needed. For all types of HCC, there is no reasonable explanation for the inconsistent diagnostic accuracy of AFP and DCP 11,12; but the different backgrounds of liver disease may be one of the main reasons.

The AUC is the most frequently used parameter for measurement of diagnostic accuracy. AUC values range from 0.5 (indicating an accuracy equivalent to that expected by chance) to 1 (denoting perfect accuracy). ${ }^{15}$ In the present study, AFP and DCP had diagnostic accuracy for NBNC HCC with AUC >0.5, but DCP seemed to be considerably better than AFP (0.853 vs. 0.763). The combination of AFP and DCP exhibited the best diagnostic accuracy (AUC $=0.867$ ). Overall, the AUC value led to conclude that combination of the two biomarkers could improve their diagnostic value, and that a combination of AFP and DCP was reliable for the diagnosis of NBNC HCC.

As this research was studied in a HBV-endemic area, the percentage of $\mathrm{HBcAb}$-positivity in patients undergoing curative resection with NBNC HCC reached $\sim 80 \%$. The pathogenesis of NBNC HCC is not known. It was thought that the risk of HCC increased sharply in response to chronic liver damage at the fibrosis stage. 5 In our study, most of the NBNC HCC cases did not diagnosed as fibrosis by pathological examination after surgery. The impact of HBcAb-positivity on the pathogenesis of NBNC HCC has been inconsistent (and even conflicting) in studies. Chronic HBV infection was considered to be the key determinant in most high-risk $\mathrm{HCC}$ areas (e.g., China, Eastern Africa) ${ }^{4}$ and HBcAbpositivity had also been considered to be a promotion factor for the development of NBNC HCC,8,16,17 according to gene-integration studies. ${ }^{18-21}$

One study found that $\mathrm{HBCAb}$-positive NBNC HCC had more advanced tumors than overt HBV (hepatitis B surface antigen positive) $\mathrm{HCC}$, but their prognosis was relatively comparable. ${ }^{22}$ Nevertheless, other scholars have found that NBNC HCC patients with HBCAbpositive have better prognosis than NBNC HCC patients with $\mathrm{HBV} / \mathrm{HCV}$-infected or HBcAb-negative. 6,23

Given the high percentage of NBNC HCC patients with $\mathrm{HBcAb}$-positivity and the conflicting opinions of studies on the effect of HBcAb, currently studies have not yet made any special discussion for the serum biomarkers for HBcAb-positivity NBNC HCC. In this study, the diagnostic accuracy of serum biomarkers of $\mathrm{HBCAb}$ positive and $\mathrm{HBCAb}$-negative subgroups respectively have been explored for better exploration of pathogenesis and clinical management of HCC. In the present study, AFP and DCP showed higher diagnostic accuracy for HBcAb-negative NBNC HCC patients (especially DCP). DCP is a well-known serum biomarker of HCC because of its diagnostic value and prognostic accuracy. 24,25 This study suggested that, compared with HBcAb-positive patients, AFP and DCP had better diagnostic accuracy for HBcAb-negative patients, and that patients with previous HBV infection and HBsAgnegativity may have better survival outcomes than HBcAb-negative patients. Most scholars have considered HBV infection to be an unfavourable factor for HCC pathogenesis. ${ }^{8}$ It is found that previous HBV infection (i.e., HBcAb-positivity) and HBsAg-negativity now seemed to be favourable factors for HCC. This result is consistent with data from two studies in 2014 and 2015, which concluded that HBsAg-negative and HBcAbpositive patients had better survival outcomes than NBNC HCC patients with HBCAb-negative or patients with $\mathrm{HBV} / \mathrm{HCV}$-infection. ${ }^{6,23}$ The population with previous HBV infection (i.e., HBcAb-positivity) and HBsAgnegativity now may have fewer methylation events at the genetic level. "Pure" NBNC HCC accounted for only $20 \%$ of all NBNC cases in the present study, so more prospective studies and further research on the relationship between methylation events at the genetic level and HBV infection are needed.

\section{CONCLUSION}

Screening is also necessary in HBcAb-positive patients for early detection of HCC, especially in the male elderly with one single lesion in the liver. With high diagnostic accuracy, good reproducibility, ease of implementation, objectivity, and non-invasiveness, AFP and DCP are suitable biomarkers and their combination could complement imaging for the diagnosis of NBNC HCC.

\section{ETHICAL APPROVAL:}

The study was approved prior to initiation of the research work by the Ethics Committees of the First Affiliated Hospital, College of Medicine, Zhejiang University.

\section{PATIENTS' CONSENT:}

As a descriptive study, a waiver for informed consent was obtained from the Ethics Committees of The First Affiliated Hospital, College of Medicine, Zhejiang University and all the data from patients were analysed anonymously.

\section{CONFLICT OF INTEREST:}

Authors declared no conflict of interest.

\section{AUTHORS' CONTRIBUTION:}

$Y L, Y C$ : Contributed to the conception and design of this work and revised it critically for important intellectual content.

JC: Contributed to data analysis, approved the final version to be published and agreed to be accountable for all aspects of the work ensuring that questions related to the accuracy or integrity of any part of the work are investigated and resolved appropriately. 


\section{REFERENCES}

1. von Kockritz L, Dufour JF. Management of chronic hepatitis $C$ in 2017. Hamostaseologie 2017; 37:186-95.

2. Llovet JM, Zucman-Rossi J, Pikarsky E, Sangro B, Schwartz M, Sherman M, et al. Hepatocellular carcinoma. Nat Rev Dis Primers 2016; 2:16018.

3. Huo TI, Ho SY. Evolution of hepatocellular carcinoma: Non-B, non-C and beyond. J Gastroenterol 2020; 55:123-4.

4. Bray F, Ferlay J, Soerjomataram I, Siegel RL, Torre LA, Jemal A. Global cancer statistics 2018: GLOBOCAN estimates of incidence and mortality worldwide for 36 cancers in 185 countries. CA Cancer J Clin 2018; 68:394-424.

5. Nishikawa $\mathrm{H}$, Osaki Y. Non-B, non-C hepatocellular carcinoma (Review). Int J Oncol 2013; 43:1333-42.

6. Utsunomiya T, Shimada M, Kudo M, Ichida T, Matsui O, Izumi N, et al. Nationwide study of 4741 patients with non-B non-C hepatocellular carcinoma with special reference to the therapeutic impact. Ann Surg 2014; 259:336-45.

7. Tateishi R, Uchino K, Fujiwara N, Takehara T, Okanoue T, Seike $\mathrm{M}$, et al. A nationwide survey on non- $B$, non- $C$ hepatocellular carcinoma in Japan: 2011-2015 update. J Gastroenterol 2019; 54:367-76.

8. Koga H, Kai K, Aishima S, Kawaguchi A, Yamaji K, Ide T, et al. Occult hepatitis $B$ virus infection and surgical outcomes in non-B, non-C patients with curative resection for hepatocellular carcinoma. World J Hepatol 2017; 9:1286-95.

9. Law JH, Tan JKH, Wong KYM, Ng WQ, Tan PS, Bonney GK, et al. Does persistent anti-HBc positivity influence the prognosis of HBsAg-negative hepatocellular carcinoma? Comparative outcomes of anti-Hbc positive versus anti-Hbc negative non-B non-C HCC. HPB (Oxford) 2019; 21:242-8.

10. Oda K, Uto H, Mawatari S, Ido A. Clinical features of hepatocellular carcinoma associated with nonalcoholic fatty liver disease: a review of human studies. Clin J Gastroenterol 2015; 8:1-9.

11. Li J, Cheng ZJ, Liu Y, Yan ZL, Wang K, Wu D, et al. Serum thioredoxin is a diagnostic marker for hepatocellular carcinoma. Oncotarget 2015; 6:9551-63.

12. Li Y, Chen J. Serum des-gamma-carboxy prothrombin for diagnosis of adult primary cancer in liver. $J$ Coll Physicians Surg Pak 2019; 29:972-6.

13. Brar G, Tsukamoto H. Alcoholic and non-alcoholic steato-hepatitis: Global perspective and emerging science. J Gastroenterol 2019; 54:218-25.

14. Kim J, Kang W, Sinn DH, Gwak GY, Paik YH, Choi MS, et al. Potential etiology, prevalence of cirrhosis, and mode of detection among patients with non-B non-C hepatocellular carcinoma in Korea. Korean J Intern Med 2019. [Epub ahead of print].

15. Alemayehu D, Zou KH. Applications of ROC analysis in medical research: Recent developments and future directions. Acad Radiol 2012; 19:1457-64.

16. Lee SB, Kim KM, An J, Lee D, Shim JH, Lim YS, et al. Clinical characteristics and potential aetiologies of non-B non-C hepatocellular carcinoma in hepatitis B virus endemic area. Liver international. Liver Int 2016; 36:1351-61.

17. Midorikawa Y, Takayama T, Nakayama H, Higaki T, Moriguchi M, Moriya K, et al. Prior hepatitis B virus infection as a co-factor of chronic hepatitis $C$ patient survival after resection of hepatocellular carcinoma. BMC Gastroenterol 2019; 19:147.

18. Kimura T, Kobayashi A, Tanaka N, Sano K, Komatsu M, Fujimori $\mathrm{N}$, et al. Clinicopathological characteristics of non-B non-C hepatocellular carcinoma without past hepatitis $B$ virus infection. Hepatology research. Hepatol Res 2017; 47:405-18.

19. Tanaka H, Iwasaki Y, Nouso K, Kobayashi Y, Nakamura S, Matsumoto $\mathrm{E}$, et al. Possible contribution of prior hepatitis $\mathrm{B}$ virus infection to the development of hepatocellular carcinoma. J Gastroenterol Hepatol 2005; 20:850-6.

20. Cacciola I, Pollicino T, Squadrito G, Cerenzia G, Orlando ME, Raimondo G. Occult hepatitis B virus infection in patients with chronic hepatitis C liver disease. N Engl J Med 1999; 341:22-6.

21. Cho EJ, Kwack MS, Jang ES, You SJ, Lee JH, Kim YJ, et al. Relative etiological role of prior hepatitis $B$ virus infection and nonalcoholic fatty liver disease in the development of non- $B$ non-C hepatocellular carcinoma in a hepatitis B-endemic area. Digestion 2011; 84(Suppl 1):17-22.

22. Wu SS, Shan QY, Xie WX, Chen B, Huang Y, Guo Y, et al. Outcomes after hepatectomy of patients with positive $\mathrm{HBcAb}$ non-B non- $C$ hepatocellular carcinoma compared to overt hepatitis B virus hepatocellular carcinoma. Clin Transl Oncol 2019 [Epub ahead of print].

23. Omichi K, Shindoh J, Yamamoto S, Matsuyama Y, Akamatsu N, Arita $\mathrm{J}$, et al. Postoperative outcomes for patients with non-B non-C hepatocellular carcinoma: A subgroup analysis of patients with a history of hepatitis B infection. Ann Surg Oncol 2015; 22(Suppl 3):S1034-40.

24. Zhang YS, Chu JH, Cui SX, Song ZY, Qu XJ. Des-gammacarboxy prothrombin (DCP) as a potential autologous growth factor for the development of hepatocellular carcinoma. Cell Physiol Biochem 2014; 34:903-15.

25. Kang SH, Kim DY, Jeon SM, Ahn SH, Park JY, Kim SU, et al. Clinical characteristics and prognosis of hepatocellular carcinoma with different sets of serum AFP and PIVKA-II levels. Eur J Gastroenterol Hepatol 2012; 24:849-56. 